\title{
Positive trials in ischaemic stroke reported at ESOC 2018
}

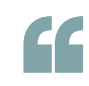

\section{imaging}

can be used to identify patients who are eligible for thrombolysis

The WAKE-UP trial, led by Götz
Thomalla and Christian Gerloff,
was designed to determine whether
MRI can be used to inform the
acute management of patients with
an unknown time of stroke onset.
Currently, these patients are ineligible
for treatment with intravenous
thrombolysis because guidelines
recommend this treatment only
when administration within $4.5 \mathrm{~h}$
of known stroke onset is possible.
The trial builds on previous work
that showed that comparison of
diffusion-weighted imaging (DWI)
and fluid attenuation inversion
recovery (FLAIR) imaging can
identify a recent stroke.
"If theress mismatch between a
visible ischaemic lesion on DWI but
no marked hyperintensity on FLAIR

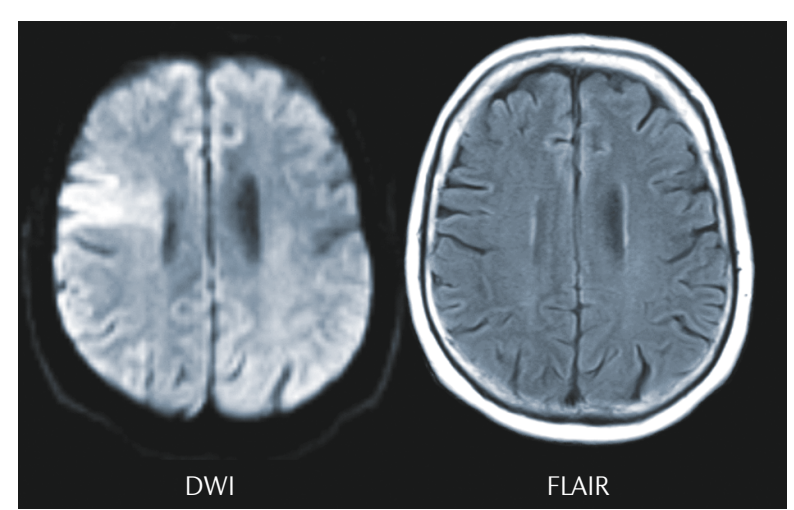

Credit: The imaging mismatch between diffusion-weighted imaging (DWI) and fluid attenuation inversion recovery (FLAIR) imaging that indicated a probable benefit of thrombolysis in the WAKE-UP trial. Image courtesy of Götz Thomalla. imaging, we can assume that the stroke has occurred recently and the patient is likely to benefit from thrombolysis," Thomalla explained at the ESOC.

The trial included 503 patients with an unknown time of stroke onset who were last known to be healthy more than $4.5 \mathrm{~h}$ previously, and who exhibited an imaging mismatch. Patients were randomly assigned to receive the tissue plasminogen activator alteplase or a placebo.

At 90 days, clinical outcomes were assessed to determine the proportion of patients who had achieved a favourable outcome, defined as a score of 0 or 1 on the modified Rankin scale. Treatment with alteplase was associated with a significantly higher likelihood of a favourable outcome than was administration of the placebo, indicating that imaging can be used to identify patients who are eligible for thrombolysis.

"This is the first positive trial of intravenous thrombolysis in patients with unknown time of symptom onset," said Thomalla. "It opens up a window for effective treatment of a large group of patients who are currently excluded from treatment."

The POINT trial focused on stroke prevention and tested whether dual antiplatelet therapy with clopidogrel and aspirin is more effective than aspirin alone in reducing the risk of recurrent stroke in the first 3 months after a transient ischaemic attack (TIA) or a minor ischaemic stroke.

This question had also been addressed in the previously published CHANCE trial, which indicated a benefit of the dual treatment. However, the design of this study left doubts about the global relevance of the findings.
"We felt it was important to continue with POINT even though CHANCE was positive because CHANCE was purely done in China and patients were treated ... differently than they would have been in Europe or the US, raising concerns about the generalizability," explained lead investigator of the POINT trial, Clay Johnston, talking at the ESOC.

The POINT trial included 4,881 patients from North America, Europe, Australia and New Zealand. Patients were enrolled within $12 \mathrm{~h}$ of a TIA or minor ischaemic stroke and randomly assigned to receive either clopidogrel and aspirin or aspirin and a placebo for 90 days.

Over the 90 days, the risk of a major ischaemic event was $6.5 \%$ among the patients who received aspirin and a placebo, and just 5\% among the patients who received clopidogrel and aspirin. This difference was significant, but was accompanied by an increase in the risk of haemorrhage that was also significant. However, the risk of haemorrhage was still small ( $0.9 \%$ with clopidogrel and aspirin compared with $0.4 \%$ with aspirin and placebo), and most of the haemorrhages were gastrointestinal and therefore treatable.

"There was no difference in fatal haemorrhage, intracranial haemorrhage or in haemorrhagic stroke," said Johnston. "To me, the trade-off is clear."

ORIGINAL ARTICLES Thomalla, G. et al. MRIguided thrombolysis for stroke with unknown time of onset. N. Engl.J. Med. https://doi.org/10.1056/ NEJMoa1804355 (2018) | Johnston, S. C. et al. Clopidogrel and aspirin in acute ischemic stroke and high-risk TIA. N. Engl.J. Med. https://doi.org/ 10.1056/NEJMoa1800410 (2018) 